\title{
Hilbert space of wormholes
}

\author{
Luis J. Garay* \\ ESA IUE Observatory, P.O. Box 50727, E-28080 Madrid, Spain \\ and Instituto de Optica, Consejo Superior de Investigaciones Cientificas, Serrano 121, E-28006 Madrid, Spain
}

(Received 17 March 1993)

\begin{abstract}
Wormhole boundary conditions for the Wheeler-DeWitt equation can be derived from the path integral formulation. It is proposed that the wormhole wave function must be square integrable in the maximal analytic extension of minisuperspace. Quantum wormholes can be invested with a Hilbert-space structure, the inner product being naturally induced by the minisuperspace metric, in which the Wheeler-DeWitt operator is essentially self-adjoint. This provides us with a kind of probabilistic interpretation. In particular, giant wormholes will give extremely small contributions to any wormhole state. We also study the whole spectrum of the Wheeler-DeWitt operator and its role in the calculation of Green's functions and effective low-energy interactions.
\end{abstract}

PACS number(s): 04.60.+n, 98.80.Hw

\section{INTRODUCTION}

Wormholes may affect the constants of nature through low-energy effective interactions $[1,2]$. In particular, they may drive the cosmological constant to zero [2] and select general relativity as the low-energy theory of gravity among Jordan-Brans-Dicke theories [3]. In order to determine which are these interactions, it is necessary to have a well-defined Hilbert space structure for wormholes [1].

The quantum wormhole wave function is given by the path integral over all possible asymptotically Euclidean spacetimes and over all matter fields defined on them whose energy-momentum tensor vanishes at infinity; it is typically labeled by the asymptotic matter field configuration. Formally, this wave function satisfies the Wheeler-DeWitt equation and the diffeomorphism constraints. Therefore, in order to find wormhole wave functions, one can equivalently solve these equations with appropriate boundary conditions, which can be derived from those for the path integral formulation [4,5]. Since spacetime is asymptotically Euclidean, the wave function will be exponentially damped for large three-geometries. On the other hand, for small three-geometries no singularities are expected and, therefore, the wave function should be regular at these configurations in some sense, so that the absence of singularities be properly reflected [4]. At least in the minisuperspace models studied so far, the wave functions calculated via path integrals are regular at every configuration in minisuperspace and, in particular, at the configurations that represent a zero volume three-geometry [6]. This expresses the fact that, although the three-geometry degenerates, the four-geometries under consideration are perfectly regular. The existence, in these circumstances, of three-geometries with a zero volume is due to the slicing procedure that has been carried

*Electronic address: Garay@cc.csic.es out in spacetime. Therefore, the wormhole wave function must be regular at any field configuration and any three-geometry. Finally, as happens in ordinary quantum mechanics, the wave function must vanish at infinite field values, since these configurations cannot dominate the wave function. All these conditions suggest that the wave function must be square integrable in superspace.

Because of the well-known difficulties in studying superspace, we shall concentrate in minisuperspace. Although the generalization of the results thus obtained to full superspace is not straightforward at all, it may be expected that some qualitative results will still hold. In minisuperspace, the Euclidean action takes the form

$$
I=\int_{0}^{\infty} d \tau N\left(\frac{1}{N^{2}} f_{\mu \nu} \dot{q}^{\mu} \dot{q}^{\nu}+V(q)\right)
$$

where $q^{\mu}(\mu=1,2, \ldots, n)$ are the degrees of freedom that represent the three-geometry and the matter fields; $N$ is the lapse function that ensures the invariance under time reparametrizations; $f_{\mu \nu}$ is the metric in minisuperspace, so that the line element in minisuperspace is

$$
d \mathcal{S}^{2}=\frac{1}{N} f_{\mu \nu} d q^{\mu} d q^{\nu}
$$

and $V(q)$ is the Wheeler-DeWitt potential, which does not contain any time derivatives $\dot{q}^{\mu}$. For each choice of the lapse function, there is a different metric in minisuperspace, all these metrics being related by conformal transformations. Therefore, for each of these choices, the quantization process will be different. However we will argue that physical results are independent of this gauge choice. Although the minisuperspace metric $f_{\mu \nu}$ has a hyperbolic signature $(-+\cdots+)$, this does not mean that there exists a time variable in minisuperspace, since superspace posseses no timelike Killing vectors. In fact, wormhole boundary conditions do not give any privilige at all to any of the minisuperspace variables.
1710

(c) 1993 The American Physical Society 
In this paper, we analyze the wormhole boundary conditions and give them a precise meaning (Sec. II). Section III is devoted to the study of the wormhole Hilbert space. We analyze the expression for the low-energy effective interaction caused by wormholes in view of this structure. Also in this section, the Green's functions of the Wheeler-DeWitt operator are considered in relation to the full spectrum of this operator. The minisuperspace models obtained by minimally or conformally coupling a massless homogeneous scalar field to a homogeneous and isotropic spacetime illustrate the Hilbert-space structure of wormholes (Sec. IV). We summarize and conclude in Sec. V.

\section{BOUNDARY CONDITIONS}

The square integrability wormhole boundary condition in minisuperspace ensures that the wormhole wave function is damped for large values of the three-volume and the matter fields. It also implies regularity of the wave function in the whole minisuperspace except on its boundary. In particular, it does not imply that the wave function is regular when the three-volume is vanishingly small. This fact leads us to study the boundary $\partial \Omega$ of the minisuperspace $\Omega$. According to Vilenkin [7], we shall define it as the set of all configurations that are singular in a general sense, i.e., such that make the metric $f_{\mu \nu} / N$ singular. On the one hand, $\partial \Omega$ may contain configurations whose singularities are due to the slicing procedure of a regular Euclidean wormhole four-geometry but that are not truly singular. This part of the boundary will be called the regular boundary. In particular, the configurations associated with a vanishing three-volume belong to the regular boundary. On the other hand, the remaining part of the boundary, the so-called singular boundary, will consist of real singularities in minisuperspace, which will not be due to the spacetime foliation. It should be mentioned that there may exist singular threegeometries that cannot be embedded in any regular fourgeometry. However, they will not appear when a slicing of a regular four-geometry is performed, and therefore they will not be relevant to the quantization procedure.

The square integrability boundary condition concerns the singular boundary, but it says nothing about the regular boundary. In this respect, two questions arise concerning the regular boundary: (i) Can we properly impose wormhole boundary conditions on it? (ii) Is it a real boundary of minisuperspace? The answer to both questions is negative. The Wheeler-DeWitt operator is hyperbolic and, for this kind of operator, the boundary value problem is well posed only if boundary conditions are imposed on characteristic surfaces of the configuration space [8], that is, on surfaces $u(q)=0$ such that their normal vectors are null:

$$
f^{\mu \nu} \nabla_{\mu} u \nabla_{\nu} u=0
$$

We will see that the regular boundary is not a characteristic surface in general (this will be the case, for in- stance, in the minisuperspace model with a conformal scalar field of Sec. IV). This question is closely related to whether the Wheeler-DeWitt operator is self-adjoint or not. In fact, the Wheeler-DeWitt operator is essentially self-adjoint and an appropriate choice of boundary conditions will determine its self-adjoint extension, provided that the boundary value problem is well posed. With respect to the second question, we have seen that the regular boundary is made of "coordinate singularities" rather than true singularities. It plays a similar role to the Schwarzschild horizon in black hole physics. It may be preventing us from gaining access to other regions of the configuration space.

For the reasons explained above, one is naturally led to consider the maximal analytic extension [9] $\bar{\Omega}$ of minisuperspace as the configuration space in which the quantization procedure can be properly accomplished. We can now state the wormhole boundary conditions as saying that the wormhole wave functions must be square integrable in the maximal analytic extension of minisuperspace. In other words, the Hilbert space of wormholes $\mathcal{W}^{0}$ consists of all normalizable solutions of the WheelerDeWitt equation

$$
\mathcal{W}^{0}=\left\{\Psi(\bar{q}) \in L_{\bar{f}}^{2}(\bar{\Omega}), \quad \hat{\bar{H}} \Psi(\bar{q})=0\right\},
$$

where the inner product is given by the natural measure in the extended minisuperspace $d \bar{q} \sqrt{\bar{f}}$, the overbar denotes quantities in the extended minisuperspace and $\bar{f}$ is the determinant of the extended metric.

This boundary condition gives full meaning to the wormhole boundary conditions formulated previously. Indeed, the boundary of the maximal extension of minisuperspace is truly singular and therefore the wormhole wave function will vanish there by virtue of square integrability. In addition, the regular boundary of minisuperspace (and in particular the configurations that represent vanishing three-geometries) is in the interior of the extended minisuperspace, and thus no additional boundary conditions are required there. Regularity of the wave function in these configurations is automatically guaranteed. As mentioned above, this boundary condition manifestly shows the absence of a spacetime character in minisuperspace and, in particular, the nonexistence of a time variable despite the Lorentzian signature of the metric in minisuperspace. In this sense, the WheelerDeWitt operator is like the Hamiltonian of a Schrödinger equation rather than a Klein-Gordon operator.

In the examples that we will consider, the WheelerDeWitt operator $\hat{\bar{H}}$, defined on the extended minisuperspace, is self-adjoint in $L_{\bar{f}}^{2}(\bar{\Omega})$, but this may not be the general case. The boundary of the extended minisuperspace consists of true singularities of the minisuperspace metric. These singular configurations are associated with true singularities of the four-geometry and, in particular, the boundary of $\bar{\Omega}$ will contain the configurations for which the three-geometry is asymptotically large. Therefore, the wave function must vanish in that boundary. In general, extended minisuperspace variables will run along the whole real line and this boundary will be at infinity. In this case, the Wheeler-DeWitt operator will 
be self-adjoint in $L_{\bar{f}}^{2}(\bar{\Omega})$. If any of the characteristic surfaces that define the boundary of minisuperspace is not located at infinity, an additional boundary condition in that surface will be necessary, so that $\hat{\bar{H}}$ be self-adjoint. The square integrability of the wave function will not be sufficient, and we will have to explicitly impose the condition that it vanishes in such a surface. In the minisuperspace models that we have considered, the square integrability condition is sufficient.

\section{HILBERT-SPACE STRUCTURE}

In order to acquire a deeper understanding of this Hilbert-space structure, let us suspend the Hamiltonian constraint for a while. Let us define an inner product in the space $\mathcal{W}$ of functions in $\bar{\Omega}$ that satisfy wormhole boundary conditions (note that these functions need not be anihilated by the Wheeler-DeWitt operator $\hat{\bar{H}}$ ) as

$$
\left(\Psi_{2}, \Psi_{1}\right)=\int_{\bar{\Omega}} d \bar{q} \sqrt{\bar{f}} \Psi_{2}^{*}(\bar{q}) \Psi_{1}(\bar{q})
$$

so that $\mathcal{W}=L_{\bar{f}}^{2}(\bar{\Omega})$. The Wheeler-DeWitt operator is self-adjoint in this inner product as already discussed. The boundary conditions in the characteristic surfaces located at a finite distance cancel the surface terms, which appear when an integration by parts is performed in the difference

$$
\left(\Psi_{2}, \hat{\bar{H}} \Psi_{1}\right)-\left(\hat{\bar{H}} \Psi_{2}, \Psi_{1}\right)
$$

The spectrum of the Wheeler-DeWitt operator, $\sigma(\hat{\bar{H}})$, will be real and will consist of an essential part and a discrete one. Let $\Psi_{\omega, \xi}(\bar{q})$ be its eigenfunctions:

$$
\hat{\bar{H}} \Psi_{\omega, \xi}(\bar{q})=\omega \Psi_{\omega, \xi}(\bar{q}),
$$

where $\omega \in \sigma(\hat{\bar{H}})$ and the index $\xi$ distinguishes between different elements of an orthonormal basis of the subspace $\mathcal{W}^{\omega}$ of eigenfunctions associated to the eigenvalue $\omega$, provided that the multiplicity of $\omega$ is different from unity. Then the set $\left\{\Psi_{\omega, \xi}(\bar{q})\right\}$ forms an orthonormal basis of $\mathcal{W}$; i.e., its elements satisfy the conditions

$$
\begin{gathered}
\left(\Psi_{\omega, \xi}, \Psi_{\omega^{\prime}, \xi^{\prime}}\right)=\delta\left(\omega-\omega^{\prime}\right) \delta\left(\xi-\xi^{\prime}\right), \\
\int d \omega d \xi \Psi_{\omega, \xi}^{*}(\bar{q}) \Psi_{\omega, \xi}\left(\bar{q}^{\prime}\right)=\frac{1}{\bar{f}} \delta\left(\bar{q}-\bar{q}^{\prime}\right),
\end{gathered}
$$

where the first relation expresses the orthonormal character of the eigenfunctions and the second is the spectral decomposition of the identity in terms of eigenfunctions of the Wheeler-DeWitt operator. For the sake of simplicity in the notation, $d \omega d \xi$ represents the measure in $\sigma(\hat{\bar{H}})$ provided by the spectral theorem for unbounded operators [10]. In particular, if the spectrum of $\hat{\bar{H}}$ is discrete, we will have to substitute $d \omega$ by $d \omega \delta\left(\omega-\omega_{n}\right), \omega_{n}$ being the discrete eigenvalues; that is, the integral will be transformed into a discrete sum.
By means of the isomorphism

$$
\begin{aligned}
\sigma: \mathcal{W} & \rightarrow \mathcal{H} \\
\Psi & \mapsto|\psi\rangle,
\end{aligned}
$$

we can assign to each basis element a state $|\omega, \xi\rangle=$ $\sigma \Psi_{\omega, \xi}(\bar{q})$, so that $\mathcal{H}$ is the set of all states $|\psi\rangle$ that are linear combinations of the basis elements

$$
|\psi\rangle=\int d \omega d \xi \psi(\omega, \xi)|\omega, \xi\rangle
$$

in which the coefficients are square integrable, i.e.,

$$
\int d \omega d \xi|\psi(\omega, \xi)|^{2}=1
$$

The space $\mathcal{H}^{0}$, of states that satisfy the Wheeler-DeWitt equation, is a subspace of $\mathcal{H}$ associated with the eigenvalue $\omega=0$ of the operator $\hat{\bar{H}}$. If the zero eigenvalue does not belong to the discrete spectrum of the WheelerDeWitt operator, $\mathcal{H}^{0}$ will not be a true subspace of $\mathcal{H}$, and therefore it will not represent the Hilbert space of wormhole wave functions. Indeed, if $0 \in \sigma_{\text {ess }}(\hat{\bar{H}})$, then no state $|0, \psi\rangle \in \mathcal{H}^{0}$ will be normalizable, since $\langle 0, \psi \mid 0, \psi\rangle=\delta(\omega=0)$. In all minisuperspace models that we have studied and those that have appeared in the literature (see, for example, Refs. $[1,4,5,11-14]$ ), the WheelerDeWitt operator has a discrete spectrum $\sigma_{\text {disc }}(\hat{\bar{H}})$ so that $0 \in \sigma_{\text {disc }}(\hat{\bar{H}})$. It should be noted that the operators $\hat{\bar{q}}^{\mu}$, which represent the three-geometry and the matter fields, are self-adjoint in $\mathcal{H}$. The spectrum of $\hat{\bar{q}}^{\mu}$ is continuous and therefore the eigenstates $|\bar{q}\rangle$ do not belong, strictly speaking, to $\mathcal{H}$ but they form a continuous basis of $\mathcal{H}$. However, since $\hat{\bar{H}}|\bar{q}\rangle \neq 0$, the vectors $|\bar{q}\rangle$ do not belong to $\mathcal{H}^{0}$, not even in the sense of states associated with continuous eigenvalues.

\section{A. The Schrödinger equation}

In quantum cosmology, the suspension of the WheelerDeWitt equation, the constraint that guarantees the invariance under time reparametrizations, gives rise to an Schrödinger equation. The wave function can be written as a path integral between two three-surfaces: In one of them we define the arguments of the wave function, while in the other we impose boundary conditions. This integral contains a sum over all possible lapse functions. The invariance of the action under reparametrizations that do not affect to these three-surfaces allow us to divide the whole set of lapse functions in equivalence classes. It is necessary to introduce a condition that fixes the gauge in this sum, so that equal contributions be counted only once. If we have two three-surfaces, this condition can be written in the form $[15,16]$

$$
\dot{N}=0 \text {. }
$$

Any other history $N(\tau)$ can be transformed into one that satisfies the gauge condition. The final form of the wave 
function is then

$$
\Psi^{0}(\bar{q})=\int_{\gamma} d N \Psi(\bar{q}, N)
$$

where the functional integral over all histories $N(\tau)$ has been substituted, due to the gauge fixing condition, by an ordinary integral along a contour $\gamma$ in the complex $N$-plane for which the integral is convergent and

$$
\Psi(\bar{q}, N)=\int \mathcal{D} \bar{q} \mathcal{D} \bar{p} \exp \left\{-\int_{0}^{N} d \tau\left(\bar{p}_{\mu} \dot{\bar{q}}^{\mu}-\bar{H}\right)\right\}
$$

The function $\Psi(\bar{q}, N)$ satisfies the Euclidean Schrödinger equation

$$
\hat{\bar{H}} \Psi(\bar{q}, N)=-\partial_{N} \Psi(\bar{q}, N),
$$

and therefore $\Psi^{0}(\bar{q})$ verifies the Wheeler-DeWitt equation $\hat{\bar{H}} \Psi^{0}(\bar{q})=0$, provided that the contour $\gamma$ is invariant under reparametrizations as when, for instance, it is closed or infinite [17].

When the boundary conditions are of the wormhole type, one of the surfaces is taken to infinity. Then the gauge-fixing condition is stronger [6]:

$$
N=1 \text {. }
$$

Indeed, this condition determines the existence of one single equivalence class under gauge transformations. Then the wormhole wave function is

$$
\Psi^{0}(\bar{q})=\lim _{|T| \rightarrow \infty} \Psi(\bar{q}, T)
$$

where $T$ is the coordinate time distance between both surfaces and

$$
\Psi(\bar{q}, T)=\int \mathcal{D} \bar{q} \mathcal{D} \bar{p} \exp \left\{-\int_{0}^{T} d \tau\left(\bar{p}_{\mu} \dot{\bar{q}}^{\mu}-\bar{H}\right)+\text { s.t. }\right\} .
$$

The surface terms that appear in this expression are due to the wormhole boundary conditions and were discussed in detail in Ref. [6]. As happens in quantum cosmology, $\Psi(\bar{q}, T)$ satisfies the Euclidean Schrödinger equation. However, the Wheeler-DeWitt equation is not obtained as a consequence of the integration over $T$, but by taking the limit $T \rightarrow \infty$, i.e., driving the surface in which boundary conditions are defined to its original place: the asymptotic region. Indeed, the function $\Psi(\bar{q}, T)$ can be written as a linear combination of eigenfunctions of $\hat{\bar{H}}$ :

$$
\Psi(\bar{q}, T)=\int d \omega d \xi \psi(\omega, \xi) e^{-\omega T} \Psi_{\omega, \xi}(\bar{q}) .
$$

If $\psi(\omega, \xi) \neq \psi(\xi) \delta(\omega)$, then terms with $\omega>0$ will not contribute in the limit $T \rightarrow \infty$ but terms with $\omega<0$ will give an infinite contribution (the situation is reversed in the limit $T \rightarrow-\infty$ ). Thus for the wormhole wave function $\Psi^{0}$ to be well defined, the only contribution that can survive is $\omega=0$, that is, the Wheeler-DeWitt oper- ator must anihilate the wave function $\Psi^{0}, \hat{\bar{H}} \Psi^{0}(\bar{q})=0$. This heuristic argument makes use of one of the main features of quantum gravity: the unboundedness from below of the gravitational action. In the Hamiltonian formulation, this feature manifests itself in the fact that the Wheeler-DeWitt operator is hyperbolic and therefore it is not bounded from below, so that it admits arbitarily negative eigenvalues.

Both in quantum cosmology and wormholes, the Wheeler-DeWitt equation is satisfied due to the reparametrization invariance, which enforces a sum over all lapse functions. However, because of the different nature of the boundary conditions in both situations, the way of performing this sum is different. In quantum cosmology, we have to sum over all possible time separations. In turn, in wormhole physics, there is only one time separation, whose value is infinity, between the surface in which the arguments of the wave function are defined and the surface in which we impose the boundary conditions [6].

It is not easy to find a physical interpretation for this Schrödinger equation. Formally, it determines the evolution of the wave function in a time which is not observable, since all observable physical quantities are expressed as sums over all possible times. However, the spectral theory of the Wheeler-DeWitt operator gives information, not only about wormholes, but also about the structure of quantum cosmology itself; that is, considering off-shell configurations and thus suspending the Hamiltonian constraint, permits the calculation of Green's functions of the Wheeler-DeWitt operator. Given two configurations $\bar{q}_{1}$ and $\bar{q}_{2}$ of extended minisuperspace, the transition amplitude between them both is defined by the path integral

$$
G\left(\bar{q}_{1}, \bar{q}_{2}\right)=\int_{\gamma} d N \int_{\mathcal{C}_{12}} \mathcal{D} \bar{q} e^{-I[\bar{q}, N]},
$$

where $\mathcal{C}_{12}$ is the set of histories $\bar{q}(\tau)$ such that $\bar{q}(0)=\bar{q}_{1}$ and $\bar{q}(1)=\bar{q}_{2}$ and $\gamma$ is an integration contour in the complex $N$ plane. If $\gamma$ is infinite or closed, $G\left(\bar{q}_{1}, \bar{q}_{2}\right)$ will be a solution of the Wheeler-DeWitt equation but, if $\gamma$ is semi-infinite, then $G\left(\bar{q}_{1}, \bar{q}_{2}\right)$ will be a Green's function of the Wheeler-DeWitt operator, that is,

$$
\hat{\bar{H}}\left(\bar{q}_{1}\right) G\left(\bar{q}_{1}, \bar{q}_{2}\right)=\frac{1}{\bar{f}} \delta\left(\bar{q}_{1}-\bar{q}_{2}\right) .
$$

We can write, at least formally, the Green's function of the Wheeler-DeWitt operator as a sum over eigenstates of $\hat{\bar{H}}$ :

$$
G\left(\bar{q}_{1}, \bar{q}_{2}\right)=\int d \omega d \xi \Psi_{\omega, \xi}\left(\bar{q}_{1}\right) \frac{1}{\omega} \Psi_{\omega, \xi}^{*}\left(\bar{q}_{2}\right) .
$$

Indeed, the expression

$$
g\left(\bar{q}_{1}, \bar{q}_{2} ; N\right)=\int_{\mathcal{C}_{12}} \mathcal{D} \bar{q} e^{-I[\bar{q}, N]}
$$

is a propagator in ordinary quantum mechanics and therefore it may be written in terms of the eigenfunctions of the Hamiltonian as 


$$
g\left(\bar{q}_{1}, \bar{q}_{2} ; N\right)=\int d \omega d \xi \Psi_{\omega, \xi}\left(\bar{q}_{1}\right) e^{-\omega N} \Psi_{\omega, \xi}^{*}\left(\bar{q}_{2}\right)
$$

The integration over $N$ along a semi-infinite contour $\gamma$ in which the integral converges gives the expected result.

\section{B. The effective interaction}

In the study of the efects that wormholes exert on the low-energy physics, it is necessary to analyze the matter field propagator

$$
\left\langle 0\left|\Phi\left(x_{1}\right) \cdots \Phi\left(x_{r}\right) \Phi\left(y_{1}\right) \cdots \Phi\left(y_{s}\right)\right| 0\right\rangle
$$

between two asymptotically Euclidean regions [1]. The points $x_{1} \cdots x_{r}$ are located in one of these regions and the points $y_{1} \cdots y_{s}$ in the other. The state $|0\rangle$ represents the ordinary scattering vacuum in quantum field theory in flat spacetime. For the sake of simplicity we will denote the product $\Phi\left(x_{1}\right) \cdots \Phi\left(x_{r}\right)$ simply by $\underline{\Phi}(x)$ and similarly for the fields at the points $y_{1} \cdots y_{s}$. We can express this propagator as the path integral over all four-metrics that can be interpolated between two asymtotically Euclidean regions and over matter fields that reach the vacuum configuration in both regions:

$$
\langle 0|\underline{\Phi}(x) \underline{\Phi}(y)| 0\rangle=\int \mathcal{D} g_{\alpha \beta} \mathcal{D} \Phi \underline{\Phi}(x) \underline{\Phi}(y) e^{-I\left[g_{\alpha \beta}, \Phi\right]} .
$$

The action $I\left[g_{\alpha \beta}, \Phi\right]$ must contain the appropriate surface terms that take into account the asymptotically Euclidean character of spacetime, as discussed in Ref. [6].

In order to perform this integration, we must first eliminate all those configurations that are related by gauge transformations and that therefore leave the action unchanged. That is, we need a condition that fixes the gauge, analogous to those discussed above for the propagation between two three-geometries and for the propagation between one three-geometry and an asymptotically Euclidean region. In the latter, the gauge condition is much stronger than in the former. Indeed, when we have two finite three-metrics the path integral over lapse functions is reduced to an ordinary integral, while when one of the surfaces corresponds to an asymptotically Euclidean region the gauge condition reduces the path integral to a single term. Now, both extreme configurations are asymptotically Euclidean. There does not exist an appropriate gauge-fixing condition, since whatever may be the choice we make, there will be various transformations that connect any history with one satisfying the gauge condition, and therefore it is not possible to fix the gauge completely. We are facing an ambiguity of the Gribov type [18]. Its cause may be the insistence on eliminating all the gauge freedom with a single global condition over all the spacetime [19].

A possible way of avoiding this ambiguity consists on introducing a three-section $\Sigma$ that divides the spacetime manifold into two disconected parts, each of them containing one of the asymptotic regions. The spectral decomposition of the identity in terms of the eigenstates of the Wheeler-DeWitt operator over the surface $\Sigma$,

$$
\mathbb{1}=\int d \omega d \xi|\omega, \xi\rangle\langle\omega, \xi|
$$

allows us to factorize the propagator

$$
\langle 0|\underline{\Phi}(x) \underline{\Phi}(y)| 0\rangle=\int d \omega d \xi\langle 0|\underline{\Phi}(x)| \omega, \xi\rangle\langle\omega, \xi|\underline{\Phi}(y)| 0\rangle,
$$

where each of the factors is given by the path integral over four-metrics and matter fields whose energy-momentum tensor vanishes at infinity, anologous to that defining an on-shell wormhole wave function. Therefore, as we have seen above only terms with $\omega=0$ will give finite nonvanishing contribution, that is,

$$
\langle 0|\underline{\Phi}(x) \underline{\Phi}(y)| 0\rangle=\int d \xi\langle 0|\underline{\Phi}(x)| 0, \xi\rangle\langle 0, \xi|\underline{\Phi}(y)| 0\rangle,
$$

where the states $|0, \xi\rangle$ form a basis of the subspace $\mathcal{H}^{0}$ of wormholes, which satisfy the Wheeler-DeWitt equation [1]. This corresponds to the idea that, in order to study the effect of wormholes on the low-energy fields, one just has to introduce a complete set of on-shell wormhole states between both asymptotic regions. These states must be solutions of the Wheeler-DeWitt equation, since wormholes cannot carry energy. It is also a consequence of the dilute wormhole approximation. If the presence of other wormholes cannot be ignored, the section $\Sigma$ will not divide the spacetime manifold into two disconnected parts. Then, the density matrix formalism will be necessary [20], in which the spectrum of the Wheeler-DeWitt operator plays a central role, in a similar way to what happened in the calculation of Green's functions.

\section{The lapse function}

The quantum formulation of wormholes depends on the choice of the lapse function. Here we will analyze this dependence and see to what extent this choice may affect our results. In the more general context of quantum cosmology, the presence of this ambiguity has already been pointed out by Hawking and Page [21].

Let us write down the Friedmann-Robertson-Walker metric in the form

$$
d s^{2}=\frac{2 \mathrm{G}}{3 \pi}\left[N^{2} \mathcal{N}^{2}(a) d \tau^{2}+a^{2} d \Omega_{3}^{2}\right]
$$

where $\mathcal{N}(a)$ is a function of $a$ that determines the gauge, and $N$ is the lapse function in this gauge, which, without loss of generality, will be regarded as constant. For the sake of simplicity, we will consider only functions of the form

$$
\mathcal{N}(a)=a^{z}, \quad z \in \mathbb{R} .
$$

In particular, $z=0$ corresponds to the proper time gauge and $z=1$ to the conformal gauge. The relation between the proper time $t$ and the coordinate time $\tau$ is given by the expression $d t=N \mathcal{N}(a) d \tau$. 
Let us begin studying which is the effect of the choice of $\mathcal{N}(a)$ on the regularity condition for three-geometries of vanishing volume. The wave function must be regular at $a=0$, expressing the fact that the spacetime manifold is not singular. Then such a manifold will admit a tangent plane at the point for which $a=0$, that is, $a \sim t$ when the proper time $t$ goes to zero. In terms of the coordinate time $\tau$, this behavior will be determined by the equation $\dot{a}(\tau) \sim N \mathcal{N}(a)$. This means that

$$
\begin{gathered}
a(\tau)^{1-z} \sim(1-z) N \tau \quad(z \neq 1) \\
a(\tau) \sim \exp (N \tau) \quad(z=1) .
\end{gathered}
$$

Consequently, the point $a=0$ is labeled by an infinite negative coordinate time if $z>1$ and finite $z<1$. The case $z=1$ is the limit in which $a=0$ corresponds to an infinite negative coordinate time but with an exponential behavior and, therefore, faster than the inverse of any power. This has a simple interpretation. The function $\mathcal{N}(a)$ defines the density of the foliation in the $3+1$ formalism, that is, the number of leaves per unit of proper time. Indeed, this number is given by the ratio $d t / d \tau$ between the interleave distances in both foliations, which is precisely the function $\mathcal{N}(a)$. The foliation associated with the conformal gauge $z=1$ is a limiting case, as we have seen. The foliations associated with values of $z$ greater than unity are less dense than the conformal one, and those associated with $z$ smaller than one are more dense.

From the classical point of view, this kind of description is certainly valid. However, in the canonical quantum theory some of these functions $\mathcal{N}(a)$ are inadmissible, since they give rise to pathologies without any physical meaning. Indeed, the requirement that the WheelerDeWitt operator be self-adjoint selects the scalar product and, therefore, the measure in minisuperspace. A consistent choice of the operator ordering and the integration measure has been discussed in the preceding sections: the volume element as integration measure and the operator ordering in $\hat{H}$ such that the kinetic term has the form of the natural Laplacian in such measure. The measure will be $d a a^{2} \mathcal{N}(a)^{-1}$, or more explicitly $a d a a^{1-z}$. Taking into account that the three-metric is $q_{i k}=a^{2} \Omega_{i k}$, we can interpret $a d a$ as the analogue of the measure in the space of three-metrics $\mathcal{D} q_{i k}$. We can see then that if $z>1$, small volume metrics will be weighted by an extremely large factor, due to the low density of the foliation in that region, thus giving them an importance that they do not deserve, since zero volume configurations do not represent special points of the spacetime manifold at all.

This discussion is based on the requirement that the Wheeler-DeWitt operator be self-adjoint. In ordinary quantum mechanics this requirement guarantees that the eigenvalues of the Hamiltonian are real, since they are possible results of measurements. In our case, wormhole wave functions are annihilated by the Wheeler-DeWitt operator, and it may seem somewhat unnecessary the requirement that this operator be self-adjoint. However, a simple argument based on the boundary conditions will convince us that this is not the case. Let $\Psi_{1}^{0}$ and $\Psi_{2}^{0}$ be two arbitrary wave functions that are solutions of the Wheeler-DeWitt equation. Then,

$$
\begin{aligned}
\left(\Psi_{2}^{0}, \hat{\bar{H}} \Psi_{1}^{0}\right)- & \left(\hat{\bar{H}} \Psi_{2}^{0}, \Psi_{1}^{0}\right) \\
& =\int_{\partial \bar{\Omega}} d \bar{\sigma}^{\mu}\left(\Psi_{2}^{0 *} \bar{\nabla}_{\mu} \Psi_{1}^{0}-\Psi_{1}^{0} \bar{\nabla}_{\mu} \Psi_{2}^{0 *}\right) .
\end{aligned}
$$

For $\hat{H}$ to be self-adjoint, both surface terms must be equal. This is in fact the case, since boundary conditions define the behavior of the wave functions in the boundary of minisuperspace, $\partial \bar{\Omega}$, and this behavior is the same for them all. Thus well-defined boundary conditions guarantee the self-adjointness of the Wheeler-DeWitt operator.

We can state that the gauge choices $\mathcal{N}(a)$ that vanish too fast when the three-geometry degenerates will not produce a well-defined canonical quantum wormhole theory, since, in these cases, the minisuperspace measure will be infinite at the configurations associated with vanishing volume three-geometries, in contradiction with the meaning of the wormhole boundary conditions. From the more general point of view of the spectrum of the WheelerDeWitt operator, these gauge choices imply $\sigma_{\text {disc }}(\hat{\bar{H}})=\emptyset$ and therefore that the zero eigenvalue is in the essential spectrum, $0 \in \sigma_{\text {ess }}(\hat{\bar{H}})$, associated with non-normalizable wave functions. We will say that these gauge fixing conditions are inadmissible from the canonical quantum point of view.

It should be stressed that different choices of the function $\mathcal{N}(a)$, even though they are admissible, will give rise to different canonical theories, not because the structure of the Wheeler-DeWitt operator or the path integral that defines the wave function will change but because of the construction of the Hilbert-space in which the wormhole wave functions live, since its scalar product depends on this choice. That is, although the local laws in minisuperspace do not depend on the choice of $\mathcal{N}(a)$, the formulation of the global laws, of boundary conditions, and therefore the Hilbert-space structure do. However, qualitative results such as the absence of giant wormholes, which is discussed in the next section, survive to this ambiguity.

On the other hand, effective interactions are defined in terms of path integrals, which contain sums over all posible lapse functions. Choosing a function $\mathcal{N}(a)$ corresponds to a partial gauge fixation in these integrals, which determine the maximal analytic extension of minisuperspace. Since the gauge fixation is carried out in such a way that the path integral is independent of the gauge fixing condition, it will be possible to perform the canonical quantization in any of these gauges, provided they are admissible. The final result for the effective interaction will be independent of this choice.

\section{MINISUPERSPACE MODELS}

In this section, we will illustrate some aspects of the wormhole Hilbert-space structure in some particular 
minisuperspace models. Let us consider a FriedmannRobertson-Walker spacetime whose metric, in the conformal gauge $(\mathcal{N}(a)=a, N=1)$, can be written as

$$
d s^{2}=\frac{2 \mathrm{G}}{3 \pi} a^{2}(\tau)\left(d \tau^{2}+d \Omega_{3}^{2}\right) .
$$

As the matter content, we will consider a minimally or conformally coupled massless homogeneous scalar field.

\section{A. Minimal coupling}

The Euclidean action, when the scalar field is minimally coupled to a Friedmann-Robertson-Walker spacetime, has the form

$$
I=\int_{0}^{\infty} d \tau\left\{-\frac{1}{2} \dot{a}^{2}-\frac{1}{2} a^{2}+\frac{1}{2} a^{2} \dot{\phi}^{2}\right\},
$$

where $\phi(\tau)$ is the minimal scalar field. Therefore, the metric in minisuperspace is

$$
d \mathcal{S}^{2}=-d a^{2}+a^{2} d \phi^{2}
$$

and the boundary of minisuperspace will consist of

$$
\partial \Omega=\{(a, \phi), a=\infty \text { or } \phi=\infty \text { or } a=0\} .
$$

Although $f^{\mu \nu}$ is not well defined at $a=0$, the singular character of this configuration is due to the foliation of spacetime. Then it may be expected that it belongs to the regular boundary of minisuperspace. On the other hand, $a=\infty$ induces an infinite volume, both in minisuperspace and spacetime. This suggests that it is truly singular. This is in fact the case. The change of variables

$$
x=a \cosh \phi, \quad y=a \sinh \phi,
$$

transforms the minisuperspace metric into the Minkowski metric

$$
d \mathcal{S}^{2}=-d x^{2}+d y^{2}
$$

although only region $I$ in Fig. 1 is represented, since in these variables the minisuperspace is

$$
\Omega=\{(x, y), \quad x>|y|\} .
$$

Part of the boundary of minisuperspace $\partial \Omega$, that defined by the points such that $x=|y|$, consists of configurations that are not singular. Therefore, it seems natural to extend the range of the variables $x$ and $y$ beyond the boundary of minisuperspace. The maximal analytic extension $\bar{\Omega}$ of the minisuperspace $\Omega$ is then given by the whole Minkowski plane (Fig. 1) and constitutes the natural basic space for the quantization of this system.

The wormhole boundary conditions that have been proposed in the previous sections require that the wave function be square integrable, i.e., that $\Psi(x, y) \in$ $L^{2}\left(\mathbb{R}^{2}\right)$. A direct consequence of these conditions is that the operators $\hat{x}, \hat{y}, i \hat{p}_{x}$, and $i \hat{p}_{y}$ (the imaginary unit appears because we are dealing with Euclidean momenta)

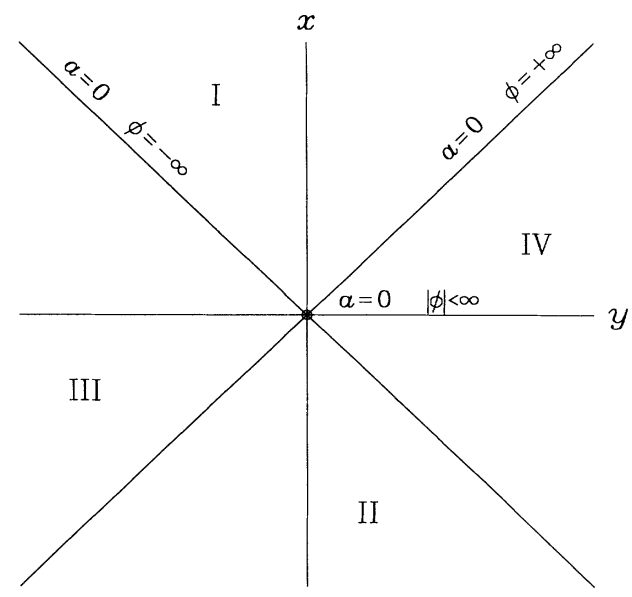

FIG. 1. Maximal analytic extension of minisuperspace corresponding to a massless scalar field minimally or conformally coupled to a Friedmann-Robertson-Walker spacetime.

are self-adjoint in their respective domains and in addition form Schrödinger pairs [10,22]. Analogously, the Wheeler-DeWitt operator

$$
\hat{\bar{H}}=-\partial_{x}^{2}+\partial_{y}^{2}+x^{2}-y^{2}
$$

is self-adjoint in $L^{2}\left(\mathbb{R}^{2}\right)$. On the other hand, the restriction to the original minisuperspace causes the indices $d_{ \pm}\left(\hat{p}_{a}\right)=\operatorname{dim}\left(\operatorname{ker}\left\{\hat{p}_{a} \pm 1\right\}\right)$ of the operator $i \hat{p}_{a}$ not to coincide and, therefore, the operator $i \hat{p}_{a}$ will not be essentially self-adjoint in $C^{\infty}\left(\mathbb{R}_{+}\right)[10,22]$. In spite of this fact, the operator $-a^{-1} \partial_{a} a \partial_{a}$ is essentially selfadjoint and consequently admits self-adjoint extensions. By means of suitable boundary conditions at $a=0$, one can choose the adecuate extension [22]. The election of this self-adjoint extension gives a precise meaning to the regularity condition proposed by Hawking and Page [4] in this model, since the self-adjointness of the WheelerDeWitt operator

$$
\hat{H}=-\frac{1}{a} \partial_{a} a \partial_{a}+\frac{1}{a^{2}} \partial_{\phi}^{2}+a^{2}
$$

is determined in this case by that of the operator $-a^{-1} \partial_{a} a \partial_{a}$. This one is a Sturm-Liouville operator that is singular at $a=0$. The most general boundary conditions that may be imposed at $a=0$ so that it be selfadjoint are of the form

$$
\lim _{a \rightarrow 0}\left\{\alpha \Psi-(\alpha \ln a-1) a \partial_{a} \Psi\right\}=0
$$

where $\alpha$ is an arbitrary parameter that determines the self-adjoint extension. To choose the value of the parameter $\alpha$, we shall look at the extended minisuperspace $\bar{\Omega}$. A basis of wave functions that are square integrable is given in Ref. [4]:

$$
\Psi_{n}^{0}(x, y)=\varphi_{n}(x) \varphi_{n}(y), \quad n=0,1,2, \ldots,
$$

where $\varphi_{n}(x)=\left(n ! 2^{n}\right)^{-1 / 2} H_{n}(x) e^{-x^{2} / 2}$ are the eigenfunctions of the Hamiltonian of the harmonic oscillator 
$-\partial_{x}^{2}+x^{2}$. If $\alpha \neq 0$, only the functions $\Psi_{n}^{0}$ for which $n$ is even will satisfy the boundary condition at $a=0$. In this case the domain of the self-adjoint extension of the Wheeler-DeWitt operator will be too small. Indeed, not only have we excluded part of the functions $\Psi_{n}^{0}$, but we have not included the wave functions $\Psi_{\phi_{0}}^{0}$, which were obtained by direct path integration in Ref. [6], either. The boundary condition defined by $\alpha=0$ is

$$
\left.a \partial_{a} \Psi(a, \phi)\right|_{a=0}=0 .
$$

Every wave function that belongs to $L_{\bar{f}}^{2}(\bar{\Omega})$ satisfies this condition as shown below. Therefore, in the restricted minisuperspace $\Omega$, the boundary conditions that the wave function be regular when the three-geometry degenerates and that it be damped for asymptotically large threegeometries can be formulated in the single boundary condition

$$
\Psi^{0}(a, \phi) \in L_{f}^{2}(\Omega) .
$$

On the other hand, the formulation in the extended minisuperspace naturally contains this condition. Indeed, the zero volume three-geometry is represented in $\bar{\Omega}=\mathbb{R}^{2}$ by the points $|x|=|y|$, i.e., by the null cone of the origin in the Minkowski metric. The normal derivatives to the null cone of the basis wave functions $\left\{\Psi_{n}^{0}(x, y)\right\}$ vanish. However, this is a condition that has naturally appeared when requiring that the wave function be square integrable in the maximal analytic extension of minisuperspace and that has not been imposed a priori. In this model, the formulation of the boundary conditions in the extended minisuperspace has allowed us to clarify the meaning of the regularity condition of the wave function in configurations of zero volume although the quantization process could also have been carried out consistently in the restricted minisuperspace. However, in other models such as the one presented below, it is necessary to consider the maximal analytic extension of minisuperspace in order to formulate the quantum theory in a consistent way, as described in the previous sections.

Apart from the discrete basis given by the wave functions $\Psi_{n}^{0}(x, y)$, there exists another continuous basis $\left\{\Psi_{k}^{0}(x, y)\right\}$ whose elements, written as a linear combinations of the discrete ones, have the form

$$
\Psi_{k}^{0}(x, y)=\sum_{n=0}^{\infty} \psi_{n}(k) \Psi_{n}^{0}(x, y)
$$

where the coefficients $\psi_{n}(k)$ are

$$
\psi_{n}(k)=\sqrt{\cosh \pi k / 2} \int d \eta \frac{\sinh ^{n} \eta}{\cosh ^{n+1} \eta} e^{-i k \eta}
$$

Since they form a continuous basis, they are not square integrable, but satisfy the closure relation

$$
\begin{aligned}
\int d x d y \Psi_{k}^{0^{*}}(x, y) \Psi_{k^{\prime}}^{0}(x, y) & =\sum_{n} \psi_{n}^{*}(k) \psi_{n}\left(k^{\prime}\right) \\
& =\delta\left(k-k^{\prime}\right) .
\end{aligned}
$$

The explicit expression of these wave functions is

$$
\Psi_{k}^{0}(x, y)=\sqrt{\cosh \pi k / 2} K_{i \frac{k}{2}}\left(\left|x^{2}-y^{2}\right| / 2\right) e^{-i k T(x, y)},
$$

where $K_{i k}(x)$ is the modified Bessel function of order $i k$ [23] and

$$
T(x, y)= \begin{cases}\operatorname{arctanh}(x / y) & \text { if }|x|<|y| \\ \operatorname{arctanh}(y / x) & \text { if }|x|>|y|\end{cases}
$$

The restriction to minisuperspace $\Omega$ acquires the simpler form, as a function of the variables $a$ and $\phi$,

$$
\Psi_{k}^{0}(a, \phi)=\sqrt{\cosh \pi k / 2} K_{i \frac{k}{2}}\left(a^{2} / 2\right) e^{-i k \phi} .
$$

The behavior of this function close to the boundaries is

$$
\begin{aligned}
\Psi_{k}^{0}(a, \phi) \sim & \frac{1}{\sqrt{k \tanh \pi k / 2}} \\
& \times\left(e^{i \theta(k)} e^{i k(\ln a-\phi)}+e^{-i \theta(k)} e^{-i k(\ln a+\phi)}\right),
\end{aligned}
$$

when $a \rightarrow 0$, where $\theta(k)$ is a real function of $k$ whose form is irrelevant, and

$$
\Psi_{k}^{0}(a, \phi) \sim \sqrt{\cosh \pi k / 2} \frac{1}{a} e^{-a^{2} / 2} e^{-i k \phi},
$$

when $a \rightarrow \infty$. Therefore, they are exponentially damped for asymptotically large three-geometries. We can see that the fact that they are not square integrable is due to its nonregular behavior in the origin, which is that of plane waves in the variables $\phi, \ln a$. However, they generate the whole space of solutions of the WheelerDeWitt equation, which are square integrable. Indeed, let

$$
\Psi^{0}(a, \phi)=\int d k \psi(k) \Psi_{k}^{0}(a, \phi)
$$

be an arbitrary linear combination such that $\psi(k) \in$ $L^{2}(\mathbb{R})$. Then

$$
a \partial_{a} \Psi^{0}(a, \phi) \sim \int d k \psi(k) \frac{k}{\sqrt{k \tanh \pi k / 2}}\left(e^{i \theta(k)} e^{i k(\ln a-\phi)}-e^{-i \theta(k)} e^{-i k(\ln a+\phi)}\right)
$$

will be a function that, in the limit $\ln a \rightarrow-\infty(a \rightarrow 0)$, will vanish, since $\psi(k)$ is square integrable.

The value $a=\sqrt{|k|}$ corresponds to the wormhole throat radius [4], since it separates the region in which the wave function decreases exponentially from the region in which it oscillates (see Fig. 2). The set of wormhole wave functions, which contain all the solutions that are square integrable, 


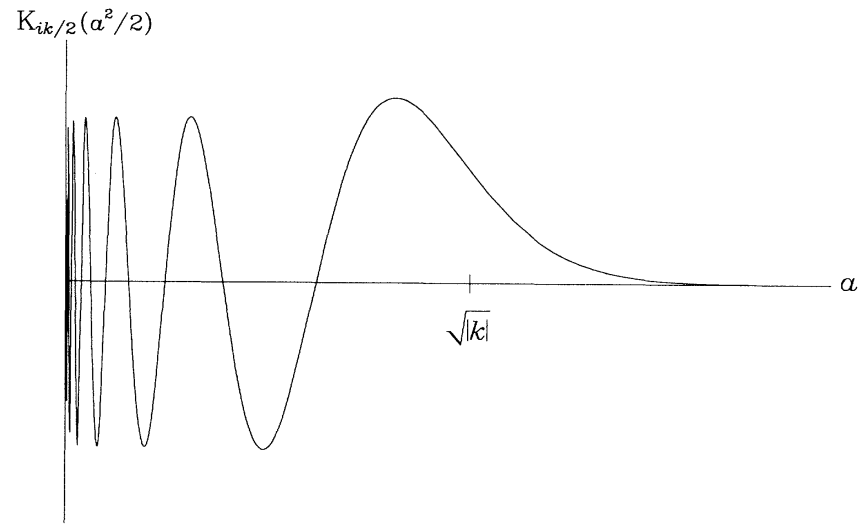

FIG. 2. Behavior of the modified Bessel function $K_{i \frac{k}{2}}\left(a^{2} / 2\right)$. The value $a=\sqrt{|k|}$ represents the wormhole throat radius.

$$
\mathcal{W}^{0}=\left\{\Psi^{0}=\int d k \psi(k) \Psi_{k}^{0}, \quad \int d k|\psi(k)|^{2}=1\right\}
$$

forms a Hilbert space which is isomorphic to $L^{2}(\mathbb{R})$. Note that this space is not $\mathcal{W}=L_{f}^{2}(\Omega)$. Indeed, neither the functions $\Psi_{k}^{0}$ nor $\Psi_{n}^{0}$ generate the whole set of square-integrable functions in $\Omega$ but only those that are eigenstates of the Wheeler-DeWitt operator with eigenvalue zero. As a consequence, it can be seen by direct calculation that they do not satisfy the closure relations of $L_{f}^{2}(\Omega)$.

If, by means of the restriction of the isomorphism $\sigma$ defined in Sec. III to $\mathcal{W}^{0}$, we associate the state $|0, k\rangle$ with the element $\Psi_{k}^{0}(x, y)$ of the basis of $\mathcal{W}^{0}$, then the coefficients of the expansion of $|0, \psi\rangle=\sigma \Psi^{0}(x, y)$ in terms of $|0, k\rangle$ will be the same as those of $\Psi^{0}(x, y)$ in terms of $\Psi_{k}^{0}(x, y)$. The scalar product in $\mathcal{H}^{0}$ is induced by $\sigma$ from the inner product in $\mathcal{W}^{0}$, in such a form that

$$
\left\langle 0, k^{\prime} \mid 0, k\right\rangle=\delta\left(k-k^{\prime}\right) .
$$

The identity operator in $\mathcal{H}^{0}$ can be decomposed into a sum of projection operators on the basis elements,

$$
\mathbb{1}_{\mathcal{H}^{0}}=\int d k|0, k\rangle\langle 0, k|
$$

which induces the following closure relation in $\mathcal{W}^{0}$ :

$$
\begin{aligned}
\mathbb{1}_{\mathcal{W}^{\circ}} \Psi^{0}\left(x_{1}, y_{1}\right)= & \sigma^{-1} \mathbb{1}_{\mathcal{H}^{\circ} \sigma} \Psi^{0}\left(x_{1}, y_{1}\right) \\
= & \int d k \Psi_{k}^{0}\left(x_{1}, y_{1}\right) \int d x_{2} d y_{2} \Psi_{k}^{0 *}\left(x_{2}, y_{2}\right) \\
& \times \Psi^{0}\left(x_{2}, y_{2}\right) .
\end{aligned}
$$

In contrast with usual procedures, it is crucial to mantain the order of integration. Indeed, if the integration over $k$ is first performed [24], an incorrect result will be obtained. This fact is closely related to the fact that there does not exist any eigenstate of the operators $\hat{x}, \hat{y}$, or equivalently, $\hat{a}, \hat{\phi}$ in $\mathcal{H}^{0}$. Indeed, although the operators $\hat{x}$ and $\hat{y}$ are self-adjoint in $\mathcal{W}=L^{2}(\bar{\Omega})$, the subspace $\mathcal{W}^{0}$ is not stable under the action of these operators. It is easily seen that if the wave function $\Psi^{0}$ satisfies the Wheeler-DeWitt equation, then the function $\left(\hat{x} \Psi^{0}\right)(x, y)=x \Psi^{0}(x, y)$ will not satisfy it, and likewise with $\left(\hat{y} \Psi^{0}\right)(x, y)=y \Psi^{0}(x, y)$.

Particularly interesting is the state $\left|0, \phi_{0}\right\rangle$ defined by

$$
\left|0, \phi_{0}\right\rangle=\int d k \psi\left(\phi_{0}, k\right)|0, k\rangle
$$

where

$$
\psi\left(\phi_{0}, k\right)=\frac{1}{\sqrt{\cosh \pi k / 2}} e^{i k \phi_{0}}
$$

or, in terms of the basis $\{|0, n\rangle\}$, by

$$
\left|0, \phi_{0}\right\rangle=\sum_{n} \psi_{n}\left(\phi_{0}\right)|0, n\rangle
$$

where

$$
\psi_{n}\left(\phi_{0}\right)=\frac{\sinh ^{n} \phi_{0}}{\cosh ^{n+1} \phi_{0}}
$$

The value $\phi_{0}$ represents the scalar field value in the asymptotic region as can be derived from the fact that the wave function $\Psi_{\phi_{0}}^{0}(x, y)=\sigma^{-1}\left|0, \phi_{0}\right\rangle$ has the form

$$
\Psi_{\phi_{0}}^{0}(x, y)=\exp \left\{-\frac{1}{2}\left(x^{2}+y^{2}\right) \cosh 2 \phi_{0}+x y \sinh 2 \phi_{0}\right\}
$$

or, in terms of the variables $a$ and $\phi$,

$$
\Psi_{\phi_{0}}^{0}(a, \phi)=\exp \left\{-\frac{1}{2} a^{2} \cosh 2\left(\phi-\phi_{0}\right)\right\} .
$$

This wave function has already been obtained as the path integral over all asymptotically Euclidean metrics and over all matter fields that reach the value $\phi_{0}$ in the asymptotic region [6].

We can introduce another continuous, orthonormal basis conjugate to $\{|0, k\rangle\}$, whose elements are

$$
|0, \chi\rangle=\int d k e^{i k \chi}|0, k\rangle
$$

which do not belong strictly to the space $\mathcal{H}^{0}$. We have seen that $\Psi_{k}^{0} \notin \mathcal{W}^{0}$ because it oscillates an infinite number of times at $a=0$. The functions $\Psi_{\chi}^{0}(a, \phi)$ are perfectly regular at $a=0$, but oscillates for large values of $a$ and $\phi$. It can be seen that

$$
\Psi_{\chi}^{0}(a, \phi)=\cos \left(\frac{1}{2} a^{2} \sinh 2(\phi-\chi)\right)+\text { terms } \in L_{f}^{2}(\Omega) .
$$

Although the operator $\hat{k}$, whose eigenstates are $|0, k\rangle$, represents both the flux through the wormhole and the 
throat radius, there does not seem to exist a simple physical interpretation for the operator $\hat{\chi}$. Nevertheless, both operators satisfy the canonical conmutation relation

$$
[\hat{k}, \hat{\chi}]=i \mathbb{1}
$$

and, in addition, form a Schrödinger pair in the realization of $\mathcal{H}^{0}$ as $L^{2}(\mathbb{R})$. The discrete basis $\{|0, n\rangle\}$ does not have a simple interpretation either. Its elements are eigenstates, corresponding to the same eigenvalue, of both the operators $\hat{H}_{x}$ and $\hat{H}_{y}$, which are formally equivalent to the harmonic oscillator Hamiltonian and that, in the space $\mathcal{W}^{0}$, acquire the form $\hat{H}_{x}=-\partial_{x}^{2}+x^{2}$, $\hat{H}_{y}=-\partial_{y}^{2}+y^{2}$, so that $\hat{\bar{H}}=\hat{H}_{x}-\hat{H}_{y}$.

The Hilbert-space structure of the set of solutions of the Wheeler-DeWitt equation with wormhole boundary conditions allow us to introduce an interpretation for the wave function. Given a wormhole in a state $|0, \psi\rangle$, the modulus squared of the product with another state $\left|0, \psi_{0}\right\rangle,\left|\left\langle 0, \psi \mid 0, \psi_{0}\right\rangle\right|^{2}$, will give an idea of the contribution of the state $\left|0, \psi_{0}\right\rangle$ to the behavior of the wormhole. In particular, $|\psi(k)|^{2}=|\langle 0, k \mid 0, \psi\rangle|^{2}$ indicates in which proportion the behavior corresponding to wormholes of radius $\sqrt{|k|}$ appears. Since $|\psi(k)|^{2} \rightarrow 0$ when $|k| \rightarrow \infty$, we can conclude that giant wormholes (of arbitrary large radius) contribute a negligible proportion, and therefore will not dominate nor fill spacetime, in agreement with the semiclassical results of Ref. [25].

The fact previously stated that the eigenstates $|x, y\rangle$ of the operators associated with the canonical variables $x$ and $y$ do not belong to $\mathcal{H}^{0}$, implies that this kind of interpretation is not applicable to quantities such as $\left|\Psi^{0}(x, y)\right|^{2}$. This can be easily understood, since the configuration $(x, y)$ is defined in a section of the fourgeometry that defines the wormhole. The wave function, however, defines global features of the whole manifold, independent of the section. It is nonsense, therefore, to talk about "a wormhole whose three-geometry is defined by $x$ and $y . "$

\section{B. Conformal coupling}

Let $\varphi(\tau)$ be a homogeneous and isotropic scalar field conformally coupled to a Friedmann-Robertson-Walker spacetime whose metric has been written in the conformal gauge. The action of the system will be

$$
I=\int d \tau\left\{-\frac{1}{2}\left(1-\varphi^{2}\right) \dot{a}^{2}-\frac{1}{2} a^{2}+\frac{1}{2} a^{2} \dot{\varphi}^{2}+a \varphi \dot{a} \dot{\varphi}\right\},
$$

so that the line element in minisuperspace is

$$
d \mathcal{S}^{2}=-\left(1-\varphi^{2}\right) d a^{2}+2 a \varphi d a d \varphi+a^{2} d \varphi^{2}
$$

and, therefore, the metric and its inverse will be

$$
f_{\mu \nu}=\left(\begin{array}{cc}
-\left(1-\varphi^{2}\right) & a \varphi \\
a \varphi & a^{2}
\end{array}\right)
$$

and

$$
f^{\mu \nu}=\left(\begin{array}{cc}
-1 & \varphi / a \\
\varphi / a & \left(1-\varphi^{2}\right) / a^{2}
\end{array}\right)
$$

respectively. The values of $a$ for which the metric or its inverse become infinite are $a=\infty$ and $a=0$. The first value corresponds to a three-geometry of infinite volume and represents a true singularity. The point $a=0$, which corresponds to a three-geometry of zero volume, is not truly singular; its singularity can be avoided by means of an analytic extension. Finally, the values of the field $\varphi= \pm 1$ are the limits in which the gravitational coupling changes its sign. However, the metric in minisuperspace is perfectly regular at these points. It might seem at first sight that the signature of $f_{\mu \nu}$ changes at these points. However, this is not the case, since there exist two vectors $n_{\mu}=(1,0)$ and $m_{\mu}=(\phi, a)$ such that $n^{2}=-1, m^{2}=1$, and $n m=0$ at every point either with $|\varphi| \leq 1$ or with $|\varphi| \geq 1$. Thus, the minisuperspace $\Omega$ will consist of all configurations $(a, \varphi)$ with $a>0$. The change of variables

$$
x=a, \quad y=a \varphi
$$

transforms the metric in minisuperspace into that of Minkowski defined in the upper semiplane, $x>0$, of Fig. 1. The maximal analytic extension is obtained by extending the range of $x$ to the whole real line so that the extended minisuperspace $\bar{\Omega}$ will be the whole plane $\mathbb{R}^{2}$ with the Minkowski metric, as happened in the case of minimal coupling. Even more, the Wheeler-DeWitt operator $\hat{\bar{H}}$ in the extended minisuperspace coincides with that of minimal coupling.

The Wheeler-DeWitt operator $\hat{H}$ in the restricted minisuperspace is not self-adjoint, although it is essentially self-adjoint. Suitable boundary conditions on the boundary $x=0$ would allow us to choose the adequate selfadjoint extension. However, because of the hyperbolic character of the Wheeler-DeWitt equation, the boundary conditions can only be imposed on characteristic surfaces (actually, they are curves, since our minisuperspace is two dimensional) which, in this model, are given by

$$
u=x+y, \quad v=x-y \text {. }
$$

Certainly, $x=0$ is not such a characteristic surface and, therefore, it does not make any sense to try to find solutions that satisfy such conditions [8]. Since the zero volume three-geometry is represented in this minisuperspace by the configuration $x=0$ with arbitrary $y$, it is not possible to give a precise meaning to the regularity condition at $a=0$ in the context of restricted minisuperspace. It is, therefore, necessary to consider its maximal analytic extension.

The quantization of this system is completly analogous to that carried out in the case of minimal coupling and the expresions given in that case are also valid here. The wormhole boundary conditions reduce to the statement

$$
\Psi^{0}(x, y) \in L^{2}\left(\mathbb{R}^{2}\right)
$$

Once more, the regularity condition at zero volume three- 
geometry is automatically satisfied, since the wave function will be regular everywhere in the extended minisuperspace $\bar{\Omega}$ and, in particular, in the region $x=0$.

The state $\left|0, \varphi_{0}\right\rangle$, which was obtained in Ref. [6] as a path integral over all asymptotically Euclidean metrics and over all matter fields whose asymptotic configuration $\varphi_{0}$ is such that the effective gravitational coupling in this region $\mathrm{G}_{\text {eff }}=\mathrm{G} /\left(1-\varphi_{0}^{2}\right)$ be positive, is annihilated by the Wheeler-DeWitt operator in the extended minisuperspace

$$
\hat{\bar{H}}\left|0, \varphi_{0}\right\rangle=0
$$

that is, $\Psi_{\varphi_{0}}^{0}(x, y)=\sigma^{-1}\left|0, \varphi_{0}\right\rangle$ satisfies the WheelerDeWitt equation. In this example we can see the close relation between the path integral and the canonical formalism. In terms of the discrete basis states $|0, n\rangle$, which are products of harmonic oscillator eigenfunctions as first given in Ref. [1], this state can be written as

$$
\left|0, \varphi_{0}\right\rangle=\sum_{n=0}^{\infty} \psi_{n}\left(\varphi_{0}\right)|0, n\rangle
$$

where

$$
\psi_{n}\left(\varphi_{0}\right)=\varphi_{0}^{n} \sqrt{1-\varphi_{0}^{2}}
$$

The norm of this vector is

$$
\left\langle 0, \varphi_{0} \mid 0, \varphi_{0}\right\rangle=\sum_{n=0}^{\infty} \varphi_{0}^{2 n}\left(1-\varphi_{0}^{2}\right)= \begin{cases}1 & \text { if }\left|\varphi_{0}\right|<1 \\ \infty & \text { if }\left|\varphi_{0}\right|>1\end{cases}
$$

Since the asymptotic region can be regarded as classical and observations can be made there, the asymptotic configurations must agree with low-energy physical predictions. In particular, the effective gravitational coupling must be positive there, i.e., $\varphi_{0}^{2}<1$. Only in this situation the state $\left|0, \varphi_{0}\right\rangle$ has any meaning [6].

\section{SUMMARY AND CONCLUSIONS}

In canonical quantum gravity, wormhole wave functions satisfy the Wheeler-DeWitt equation and the quantum constraints associated with the invariance under spatial changes of coordinates. Furthermore, they are subjected to suitable boundary conditions, which may be deduced from the path integral formulation. We have restricted ourselves to minisuperspace, where only a finite number of degrees of freedom have not been frozen out. The asymptotically Euclidean character of wormholes makes the wave function decrease exponentially for configurations that represent arbitrarily large threegeometries. Likewise, the wave function must vanish for large values of the matter fields. Also the wave function must be regular when the three-volume vanishes, since the spacetime manifold is not singular at that point. The singularity of the three-geometry is only due to the slicing procedure.

The boundary of minisuperspace consists of all those configurations that are singular in some general sense, including those mentioned above for which the minisuperspace metric is singular although it corresponds to a coordinate singularity. Therefore, it seems necessary consider the maximal analytic extension of minisuperspace as the natural configuration space for quantization. The true singularities, such as those corresponding to infinite values of the three-volume or the matter fields, belong to the boundary of extended minisuperspace and the regular configurations, including those associated with zero three-volume, belong to its interior. Then, the wormhole boundary conditions can be simply formulated in the following way: The wormhole wave functions must be square integrable in the maximal analytic extension of minisuperspace. This condition ensures that the wave function vanishes at the truly singular configurations and guarantees its regularity at any other configuration, including those which represent zero volume three-geometries. In fact, considering the maximal analytic extension of minisuperspace, we avoid the necessity of imposing boundary conditions at zero volume three-geometries that guarantee the self-adjointness of the Wheeler-DeWitt operator. This operator is hyperbolic and, therefore, in order to have a well-posed boundary value problem, boundary conditions should be imposed on characteristic surfaces of this operator. However, the surface in minisuperspace associated to vanishing three-geometries is not of this type, in general, and consequently, it is meaningless to impose boundary conditions on it.

Since wormholes are square integrable in the extended minisuperspace, they form a Hilbert space whose inner product is naturally induced by the minisuperspace metric, in which the Wheeler-DeWitt operator is essentially self-adjoint. Then we can introduce an interpretation for the wormhole wave function in terms of overlaps between different states. These overlaps give an idea of the contribution of a given state to the behavior of a wormhole in another state. In particular, we can conclude that giant wormholes should not contribute significantly to any wormhole state.

The study of the whole spectrum of the WheelerDeWitt operator is useful in the calculation of Green's functions in quantum cosmology, since they can be written in terms of a complete set of eigenfunctions of this operator. On the other hand, the evaluation of the effective wormhole interaction beyond the dilute wormhole approximation also requires the whole spectrum and not only the states that satisfy the Wheeler-DeWitt equation, that is, the eigenstates of zero eigenvalue as happens when this approximation is valid.

Finally, a consistent canonical quantum formulation of wormholes requires a restriction of the gauge fixing conditions. For instance, gauge conditions that vanish too fast with the three-volume are not admissible. The canonical quantization process contains an ambiguity, since different admissible gauge-fixing conditions give rise to different Hilbert spaces. However, physical results, such as effective interactions, are independent of the gauge con- 
dition, due to the fact that they can be given as path integrals.

\section{ACKNOWLEDGMENTS}

I wish to thank Pedro González-Díaz, Guillermo Mena Marugán and Peter Tinyakov for their valuable com- ments on the manuscript. I also thank Instituto de Matemáticas y Física Fundamental, C.S.I.C., for hospitality. This work was supported by DGICYT under Contract No. PB91-0052 and by a Basque Country Grant. The ESA IUE Observatory is affiliated with the Astrophysics Division, Space Science Department, ESTEC.
[1] S. W. Hawking, Phys. Rev. D 37, 904 (1988).

[2] S. Coleman, Nucl. Phys. B310, 643 (1988).

[3] L. J. Garay and J. García-Bellido, Nucl. Phys. B (to be published).

[4] S. W. Hawking and D. N. Page, Phys. Rev. D 42, 2655 (1990).

[5] L. M. Campbell and L. J. Garay, Phys. Lett. B 254, 49 (1991).

[6] L. J. Garay, Phys. Rev. D 44, 1059 (1991).

[7] A. Vilenkin, Phys. Rev. D 37, 888 (1988).

[8] P. Puig Adam, Ecuaciones Diferenciales (Biblioteca Matemática, Madrid, 1955).

[9] S. W. Hawking and G. F. R. Ellis, The Large Scale Structure of Spacetime (Cambridge University Press, Cambridge, England, 1973).

[10] M. Reed and B. Simon, Methods of Modern Mathematical Physics I. Functional Analysis (Academic, New York, 1972).

[11] H. F. Dowker, Nucl. Phys. B331, 194 (1990).

[12] A. Lyons, Nucl. Phys. B324, 253 (1989).

[13] H. F. Dowker and R. Laflamme, Nucl. Phys. B366, 209 (1991).
[14] A. Zhuk, Phys. Rev. D 45, 1192 (1992).

[15] C. Teitelboim, Phys. Rev. D 28, 297 (1983).

[16] J. J. Halliwell, Phys. Rev. D 38, 2468 (1988).

[17] J. J. Halliwell and J. B. Hartle, Phys. Rev. D 43, 1170 (1991).

[18] V. N. Gribov, Nucl. Phys. B139, 1 (1978).

[19] P. Ramond, Field Theory: A Modern Primer, 2nd ed. (Addison-Wesley, New York, 1990).

[20] P. F. González-Díaz, Nucl. Phys. B351, 767 (1991).

[21] S. W. Hawking and D. N. Page, Nucl. Phys. B264, 185 (1986).

[22] A. Galindo and P. Pascual, Mecánica Cuántica (Eudema, Madrid, 1989).

[23] Handbook of Mathematical Functions with Formulas, Graphs and Mathematical Tables, edited by $M$. Abramowitz and I. A. Stegun (Dover, New York, 1965).

[24] I. S. Gradshteyn and I. M. Ryzhik, Table of Integrals, Series and Products (Academic, London, 1965).

[25] S. B. Giddings and A. Strominger, Nucl. Phys. B306, 890 (1988). 